\title{
Realistic Covariance Prediction for the Earth Science Constellation
}

\author{
Matthew Duncan ${ }^{*}$ and Anne Long ${ }^{\dagger}$ \\ a.i. solutions, Inc. Lanham, MD, 20706
}

\begin{abstract}
Routine satellite operations for the Earth Science Constellation (ESC) include collision risk assessment between members of the constellation and other orbiting space objects. One component of the risk assessment process is computing the collision probability between two space objects. The collision probability is computed using Monte Carlo techniques as well as by numerically integrating relative state probability density functions. Each algorithm takes as inputs state vector and state vector uncertainty information for both objects. The state vector uncertainty information is expressed in terms of a covariance matrix. The collision probability computation is only as good as the inputs. Therefore, to obtain a collision calculation that is a useful decision-making metric, realistic covariance matrices must be used as inputs to the calculation. This paper describes the process used by the NASA/Goddard Space Flight Center's Earth Science Mission Operations Project to generate realistic covariance predictions for three of the Earth Science Constellation satellites: Aqua, Aura and Terra.
\end{abstract}

\section{Nomenclature}

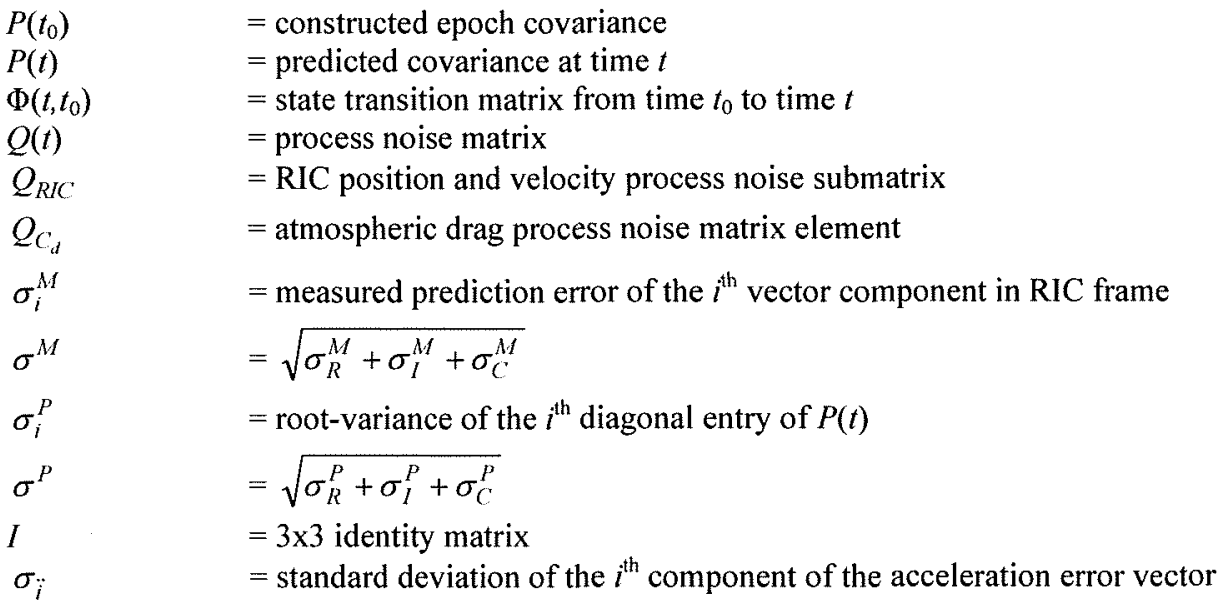

\footnotetext{
* Mission Analyst, matthew.duncan@ai-solutions.com

Chief Systems Engineer, anne.long@ai-solutions.com, AIAA Senior Member
}

1 


\section{Introduction} HE Earth Science Mission Operations (ESMO) Project at NASA/Goddard Space Flight Center (GSFC)
has established a routine conjunction assessment process for satellites in the Earth Science Constellation $(\mathrm{ESC})^{1}$. The conjunction assessment $(\mathrm{CA})$ process consists of:

1. Predicting close approaches between members of the ESC and other objects in the United States Strategic Command's (USSTRATCOM) Space Object Catalog

2. Assessing the collision risk for any close approach predictions

3. Performing any necessary risk-mitigating action

Several times a week, close approach predictions are made by Cheyenne Mountain's $1^{\text {st }}$ Space Control Squadron (SPCS) personnel. Close approach information is sent to NASA/GSFC where the GSFC CA team performs collision risk assessment analysis. One component of the risk assessment process is to compute the collision probability between objects. For the ESC, the collision probability is computed using two methods: (1) numerical integration of a relative state probability density function and (2) Monte Carlo simulations. In both cases, inputs to the probability calculation consist of state vector and state vector uncertainty information, as well as a user-specified keep-out region.

The state information provided by $1^{\text {st }}$ SPCS is generated using observations collected from the US Space Surveillance Network (SSN). The orbit determination methodology employed by Cheyenne Mountain is a weighted batch least-squares (BLS) estimation method, which provides a "best-fit" trajectory (one that best matches the observations). The covariance derived through the BLS process is often found to be optimistic ${ }^{2-4}$, since the errors in the dynamic model are usually not included in the covariance calculation. $1^{\text {st }}$ SPCS makes use of a consider parameter technique $e^{5}$, where the uncertainties of unmodeled systematic errors (i.e. errors that are not estimated through the orbit determination process) are still "considered" when the state covariance is computed. This method has the effect of adding additional state errors directly into the best-fit derived covariance matrix. The consider parameter method helps shape the epoch covariance, but the predictive covariance uncertainties are still typically underestimated when the covariance is propagated forward in time.

Current operational procedures require that $1^{\text {st }}$ SPCS provide state and covariance information for close approach predictions that violate a specified alert threshold. After this information is received at GSFC, the probability calculations can be made. In addition to using the SSN-based solutions, risk assessment and risk mitigation analyses are performed using GSFC-derived state and covariance as inputs to collision calculation. In particular, this is done for the three ESC members that comprise the Earth Observing System (EOS); namely, Aqua, Aura, and Terra. Using mission-derived data in the risk assessment process allows for an independent corroboration and assessment of the uncertainty information provided by $1^{\text {st }}$ SPCS. Moreover, realistic covariance predictions with orbit adjust maneuvers modeled can be used to probabilistically assess how each maneuver will affect a given close approach prediction.

This paper describes the process used to generate realistic covariance predictions for Aqua, Aura, and Terra. The process is relatively straightforward; the definitive covariance is propagated ahead in time using the state transition matrix $\Phi$ and process noise matrix $Q$ as follows:

$$
P(t)=\Phi\left(t, t_{0}\right) P\left(t_{0}\right) \Phi\left(t, t_{0}\right)^{T}+Q(t)
$$

To ensure that the covariance predictions accurately reflect the orbital uncertainties for a given prediction period, calibration of the prediction errors, which is achieved by adjusting the process noise variance acceleration terms, must be performed. The following sections present the details of this covariance generation process, which consists of three steps: (1) Construction of a realistic definitive covariance matrix; (2) Characterization of the orbit prediction errors; and (3) Calibration of the covariance growth rate. Numerical results for Aura are included. 


\section{The Covariance Generation Process}

This section describes the process employed by the ESMO project to construct a realistic covariance prediction for member satellites of the ESC. The definitive covariance matrix is propagated ahead in time using the state transition matrix and process noise matrix. If the predictive covariance is to accurately represent the future state uncertainty, prediction errors that arise from force modeling errors must be included. Therefore, the definitive covariance matrices and the process noise matrix are empirically calibrated to match measured position prediction errors. The following sections describe this three-step process in more detail.

\section{A. Definitive Covariance Construction}

Orbit determination for the EOS missions is performed in the GSFC Flight Dynamics Facility (FDF). The state estimation software is the Real-Time Orbit Determination $\left(\right.$ RTOD $\left.^{\circledR}\right)$ system, which uses an extended Kalman filter to process tracking measurements from NASA's space and ground networks. RTOD $^{\circledR}$ is updated daily with the latest solar flux and geomagnetic index (GMI) indices. Examination of several months of definitive covariance solutions shows that the solutions are very consistent from week to week, i.e. the estimated position uncertainty numbers vary by only a few meters. Therefore, in the absence of any orbit adjust maneuvers, it is sufficient to create a single "representative" definitive epoch covariance to be used to initialize the predictive covariance generation. The representative epoch covariance $P\left(t_{0}\right)$ is constructed by averaging the definitive RTOD $^{\otimes}$ covariance elements using the procedure described below. It was observed that the correlation terms of the definitive covariance vary significantly with time - e.g. some of the correlations are 'broken' during tracking data contacts. In particular, the correlation terms for the in-track position/radial velocity and the radial position/in-track velocity vary considerably with time. The averaging method removes these short-term variations.

Let $P_{i j}(t)$ denote the $(i, j)$ element of the definitive covariance at instant $t$. Additionally, define $\overline{P_{i j}}\left(t_{0}\right)$ as an "averaged" time-history value based on $P_{i j}(t)$ for some specified time span. The following steps are performed to construct a realistic epoch covariance matrix:

Step 1: Select a time span and compute the average value for each position/velocity variance element $P_{i i}$.

$$
\overline{P_{i i}}\left(t_{0}\right)=\frac{1}{N} \sum_{k=1}^{N} P_{i i}\left(t_{k}\right) \quad \text { for each } i=1 \ldots 6 \text { and some sample size } N \text {. }
$$

Step 2: Similar to the time series averaging performed in Step 1, compute the average correlation value for each of the off-diagonal terms. For each $(i, j)$ entry, denote the average correlation coefficient as $\overline{\rho_{i j}}\left(t_{0}\right)$.

Step 3: Using the average values computed in steps 1 and 2, compute the off-diagonal terms $\overline{P_{i j}}\left(t_{0}\right)$ as follows:

$$
\overline{P_{i j}}\left(t_{0}\right)=\overline{\rho_{i j}}\left(t_{0}\right)\left[\overline{P_{i i}}\left(t_{0}\right) \cdot \overline{P_{j j}}\left(t_{0}\right)\right]^{1 / 2}
$$

For convenience, $\bar{P}_{i j}\left(t_{0}\right)$ is abbreviated as $P\left(t_{0}\right)$ in the remaining discussion.

Steps 1-3 describe the construction of the $6 \times 6$ epoch covariance. The final size of $P\left(t_{0}\right)$ is a $7 \times 7$ matrix, where the uncertainties due to atmospheric drag are included, since atmospheric drag modeling error is the primary dynamic error contributing to the prediction error of the EOS spacecraft. The drag uncertainty is represented by the uncertainty in a unitless coefficient $C_{d}$. Thus the $6 \times 6$ covariance is augmented to include an initial $\mathrm{C}_{d}$ uncertainty by placing the initial $\mathrm{C}_{d}$ variance along the diagonal and the 7 th row and 7 th column off-diagonal terms are set to zero. That is, $P_{7 j}\left(t_{0}\right)=P_{i 7}\left(t_{0}\right)=0$ for all $\mathrm{i} \neq \mathrm{j}$. 


\section{B. Prediction Error Characterization}

In the absolute sense, state errors cannot be measured. Sensor measurements and dynamic modeling errors present in the orbit determination process lead to prediction errors when the epoch state is used in future predictions. Thus orbit prediction errors can only be approximated by examining and trending past performance. The prediction error characterization methodology employed for the EOS missions is to measure the orbit prediction errors by comparing predictive ephemerides with definitive "truth" solutions over the same time period. These differences or "overlap" comparisons are used to assess the accuracy of state predictions.

The truth trajectory is constructed by 'post-processing' the previous day's tracking data using definitive solar flux and GMI indices files. Each day all measurements received up to the current time (i.e. 12:00 GMT on the current day) are processed to create the definitive solution for the current day. Any additional tracking data that may have significantly lagged the filter's real-time processing is included as well. Each day's definitive state corresponding to 12:00 GMT is used as the basis for the predictive ephemeris generation.

Prediction error characterization consists of performing numerous overlap comparisons between predictive and definitive ephemerides over some period of time (i.e. for a specified sample size). Both position and velocity differences are calculated and are expressed in the radial, in-track, cross-track (RIC) coordinate frame. Error statistics for each RIC component, including the mean and standard deviation, are computed every 6 hours for prediction times up to 7 days. Additional statistics, such as the root-meansquare (RMS) are computed as well. The RMS, which is defined as

$$
\mathrm{RMS}^{2}=\text { mean }^{2}+\text { standard deviation }{ }^{2}
$$

is chosen to represent the 1-sigma measured errors. After the statistics are collected, error prediction polynomials are computed by fitting the RMS values in a least squares sense. In particular, a second order polynomial for each RIC component is computed, which represents the 1-sigma error value for that component as a function of time. Each component is written as

$$
\sigma_{i}^{M}=a_{i} t^{2}+b_{i} t+c_{i}
$$

where ' $M$ ' denotes the measured prediction errors for the $i^{\text {th }}$ component (position or velocity).

Since prediction errors are driven primarily by unpredicted variations in the solar activity and subsequent errors in the atmospheric drag modeling, the polynomial coefficients $\left\{a_{i}, b_{i}, c_{i}\right\}$ must be routinely re-computed. This is particularly important for the in-track uncertainty since errors in the atmospheric drag modeling manifest themselves as in-track position errors. Thus the in-track prediction errors will tend to decrease as the solar flux decreases. For the other position components, radial and crosstrack errors are primarily due to modeling errors in the Earth's geopotential and solar radiation pressure (SRP). These errors tend to be more of a constant bias and typically don't exhibit a strong 'seasonal' dependence as does drag.

\section{Predictive Covariance Calibration}

Calibration of the covariance growth rate is achieved by adjusting the process noise matrix $Q$ until the propagated root-variance, denoted by $\sigma_{i}^{P}$, matches the measured sigma values $\sigma_{i}^{M}$ over the specified prediction span. The epoch covariance $P\left(t_{0}\right)$ is propagated forward in time using the equation expressed in the mean of J2000.0 (MJ2000) inertial reference frame

$$
P(t)=\Phi\left(t, t_{0}\right) P\left(t_{0}\right) \Phi\left(t, t_{0}\right)^{T}+Q(t)
$$

The state transition matrix $\Phi\left(t, t_{0}\right)$ links the initial state at time $t_{0}$ to the state at time $t$. $\Phi$ is a matrix of partial derivatives of the state at the current time with respect to the state at the initial time. The $\Phi$ matrix is computed numerically using the central differencing method, which is accumulated using a very small step size using a high fidelity model such as that listed in Table 2. 
The process noise matrix $Q(t)$ increases the state uncertainty at each step in an additive fashion. The presence of the $Q$ matrix accounts for errors in the dynamic model, such as mismodeling of atmospheric drag, solar radiation pressure, and the Earth's geopotential. The matrix $Q$ must have the same dimensions as $\mathrm{P} . \mathrm{Q}$ is expressed in the following block diagonal form:

$$
Q(t)=\left(\begin{array}{ll}
Q_{R I C} & 0_{1 x 6} \\
0_{6 x 1} & Q_{C_{d}}
\end{array}\right)
$$

where

$$
\begin{aligned}
& Q_{R I C}=\quad \text { the process noise submatrix for the position and velocity vectors (in RIC frame) } \\
& Q_{C_{d}}=\quad \text { the process noise matrix element for the atmospheric drag parameter } \mathrm{C}_{d}
\end{aligned}
$$

The RIC process noise matrix is a $6 \times 6$ while the $\mathrm{Cd}$ ' $\mathrm{Q}$ ' is a singleton. The QRIC matrix is formulated such that the random acceleration uncertainties are directly integrated into the covariance prediction process. The RIC acceleration sigma values are denoted by $\left\{\sigma_{\ddot{R}}, \sigma_{\ddot{I}}, \sigma_{\ddot{C}}\right\}$. Then the RIC root-variances are given as:

$$
Q_{R I C}=\left(\begin{array}{cccccc}
\left(\sigma_{\ddot{R}}\right)^{2} \frac{\Delta T^{4}}{3} & 0 & 0 & \left(\sigma_{\ddot{R}}\right)^{2} \frac{\Delta T^{3}}{2} & 0 & 0 \\
0 & \left(\sigma_{\ddot{I}}\right)^{2} \frac{\Delta T^{4}}{3} & 0 & 0 & \left(\sigma_{\ddot{I}}\right)^{2} \frac{\Delta T^{3}}{2} & 0 \\
0 & 0 & \left(\sigma_{\ddot{C}}\right)^{2} \frac{\Delta T^{4}}{3} & 0 & 0 & \left(\sigma_{\ddot{C}}\right)^{2} \frac{\Delta T^{3}}{2} \\
\left(\sigma_{\ddot{R}}\right)^{2} \frac{\Delta T^{3}}{2} & 0 & 0 & \left(\sigma_{\ddot{R}}\right)^{2} \Delta T^{2} & 0 & 0 \\
0 & \left(\sigma_{\ddot{I}}\right)^{2} \frac{\Delta T^{3}}{2} & 0 & 0 & \left(\sigma_{\ddot{I}}\right)^{2} \Delta T^{2} & 0 \\
0 & 0 & \left(\sigma_{\ddot{C}}\right)^{2} \frac{\Delta T^{3}}{2} & 0 & 0 & \left(\sigma_{\ddot{C}}\right)^{2} \Delta T^{2}
\end{array}\right)
$$

where $\Delta T$ denotes the propagation step-size. For convenience, $Q_{R I C}$ is expressed in the compact form

$$
Q_{R I C}=\left(\begin{array}{cc}
\frac{\Delta T^{4}}{3} I \cdot \bar{q}_{a c c} & \frac{\Delta T^{3}}{2} I \cdot \bar{q}_{a c c} \\
\frac{\Delta T^{3}}{2} I \cdot \bar{q}_{a c c} & \Delta T^{2} I \cdot \bar{q}_{a c c}
\end{array}\right)
$$

where

$$
\bar{q}_{a c c}=\left(\begin{array}{c}
\sigma_{\ddot{R}} \\
\sigma_{\ddot{I}} \\
\sigma_{\ddot{C}}
\end{array}\right)^{2} \text { and } I=\left(\begin{array}{ccc}
1 & 0 & 0 \\
0 & 1 & 0 \\
0 & 0 & 1
\end{array}\right) \text {. }
$$

Values for $\bar{q}_{a c c}$ and $Q_{C_{d}}$ are adjusted so that the root-variances $\sigma_{i}^{P}$ of $\mathrm{P}(\mathrm{t})$ are in good agreement with the corresponding prediction error profiles $\sigma_{i}^{M}$. In particular, comparisons between the RIC position differences are made, where the elements of the process noise matrix are adjusted so that 


$$
\left|\sigma_{i}^{M}-\sigma_{i}^{P}\right|<K_{i} \quad \text { for each } i \in\{R, I, C\} \text { and some constant } K \text {. }
$$

The steps for adjusting the values $\bar{q}_{a c c}$ and $Q_{C_{d}}$ consists of:

1. choosing a set of initial values

2. generating the predictive covariance $P(t)$

3. making the comparisons described in Equation 11

4. adjusting $\bar{q}_{a c c}$ and $Q_{C_{t}}$ until the 'convergence criteria' are satisfied

Changes made to the $Q_{C_{d}}$ affect the in-track error predictions. Since errors in atmospheric drag modeling lead to errors in the in-track position, the $Q_{C_{d}}$ value will affect the predicted in-track position variance. The position variances are also directly correlated with the RIC acceleration variances; therefore changes to the $\left\{\sigma_{\ddot{R}}, \sigma_{\ddot{I}}, \sigma_{\ddot{C}}\right\}$ values directly affect their RIC position counterparts. Additionally, there exists some correlation between the in-track prediction error and the acceleration error in the radial direction. Thus adjustments made to $\sigma_{\ddot{R}}$ will have some effect on the in-track predictions as well. Table 1 summarizes how elements of $Q(t)$ map to changes in the predicted position errors when the covariance is propagated forward.

Table 1: Process Noise Effects

\begin{tabular}{|c|c|}
\hline $\boldsymbol{Q}(\boldsymbol{t})$ Element & Affected $\boldsymbol{P}(\boldsymbol{t})$ Position Error \\
\hline$\sigma_{\ddot{R}}$ & Radial, In-Track \\
\hline$\sigma_{\ddot{I}}$ & In-Track \\
\hline$\sigma_{\ddot{C}}$ & Cross-Track \\
\hline$Q_{C_{d}}$ & In-Track \\
\hline
\end{tabular}

Although both $Q_{C_{d}}$ and $\sigma_{\ddot{j}}$ will affect the in-track error prediction, only one of them is adjusted when calibrating the predicted covariance in accordance with Equation 11.

\section{Aura Case Study}

The methodology described in Section II is now applied to a specific case in which a realistic covariance prediction is made for the Aura spacecraft. First, an 'averaged' epoch covariance $P\left(t_{0}\right)$ is constructed. Second, the position prediction errors $\left\{\sigma_{R}^{M}, \sigma_{I}^{M}, \sigma_{C}^{M}\right\}$ are measured for prediction times up to 7 days. Third, the covariance prediction is calibrated by adjusting the process noise matrix $Q(t)$ until the predicted position root-variances $\left\{\sigma_{R}^{P}, \sigma_{I}^{P}, \sigma_{C}^{P}\right\}$ match the measured prediction errors.

\section{Force Modeling and Initial Conditions}

Both the state and covariance are propagated forward using FreeFlyer ${ }^{3}$. Table 2 lists the perturbative forces modeled and Table 3 lists the state vector initial conditions used in this case study.

Table 2: Aura Force Modeling

\begin{tabular}{|l|r|}
\hline Parameter & Modeling \\
\hline Earth Geopotential Model & 30x30 Joint Gravity Model (JGM) -2 \\
\hline Non-Central Bodies & Sun, Moon (JPL DE200 Ephemeris File) \\
\hline Atmospheric Density Model & Jacchia Roberts \\
\hline Solar Radiation Pressure Model & Thin plate model with reflectivity coefficient \\
& $\mathrm{C}_{\mathrm{r}}=1.4$ \\
\hline Effective Solar Radiation Pressure Area & $47.95 \mathrm{~m}^{2}$ \\
\hline Effective Drag Area & $47.95 \mathrm{~m}^{2}$ \\
\hline Numerical Integrator & Runge-Kutta 8(9) with 60-second step size \\
\hline
\end{tabular}


Table 3: Aura Initial State Conditions

\begin{tabular}{|l|r|}
\hline Parameter & Value \\
\hline State Epoch (MJ2000) & 16-Mar, 2006 13:19:20.000 \\
\hline $\mathrm{X}(\mathrm{m})$ & 435179.356 \\
\hline $\mathrm{Y}(\mathrm{m})$ & -923269.985 \\
\hline$Z(\mathrm{~m})$ & 6997996.863 \\
\hline$X \operatorname{dot}(\mathrm{m} / \mathrm{s})$ & -7079.883 \\
\hline$Y \operatorname{dot}(\mathrm{m} / \mathrm{s})$ & -2489.657 \\
\hline$Z \operatorname{dot}(\mathrm{m} / \mathrm{s})$ & 111.781 \\
\hline & \\
\hline Mass $(\mathrm{kg})$ & 3046.5 \\
\hline$C_{d}$ & 1.7 \\
\hline$C_{d}$ variance & 0.01 \\
\hline
\end{tabular}

\section{E. Definitive Covariance Construction}

The epoch covariance $P\left(t_{0}\right)$ is constructed using the averaging method described in Section II-A, where the position/velocity covariances are determined using definitive solutions spanning the time March 16, 2006 - March 24, 2006. Figure 1 and Figure 2 show the definitive RIC and total position and velocity rootvariances over the 7-day period. These figures show that there are small variations during the week, where the position uncertainty magnitude ranges between 1 and $4 \mathrm{~m}$ and the magnitude of the definitive velocity uncertainty values range between 1 and $4 \mathrm{~mm} / \mathrm{s}$.
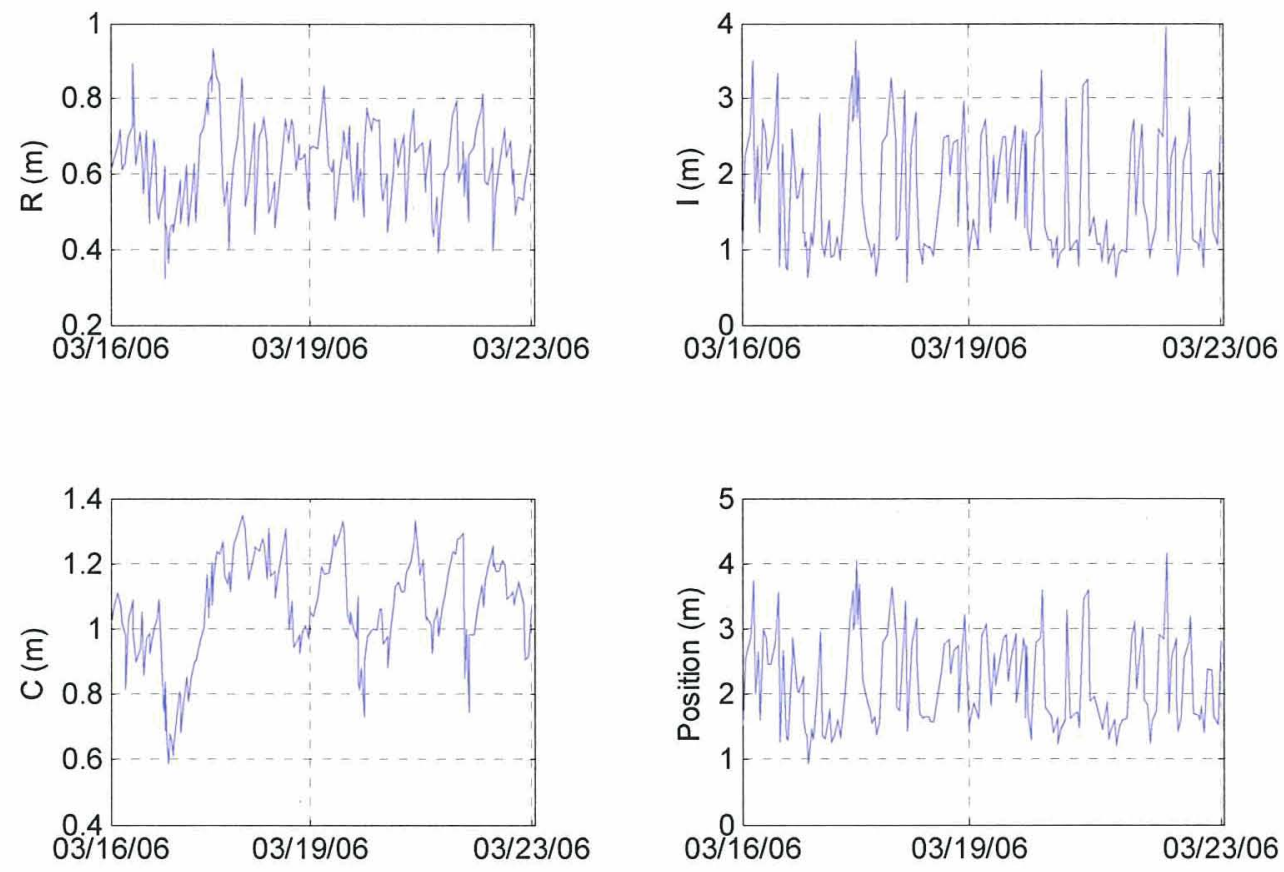

Figure 1: Definitive Position Root-Variance 

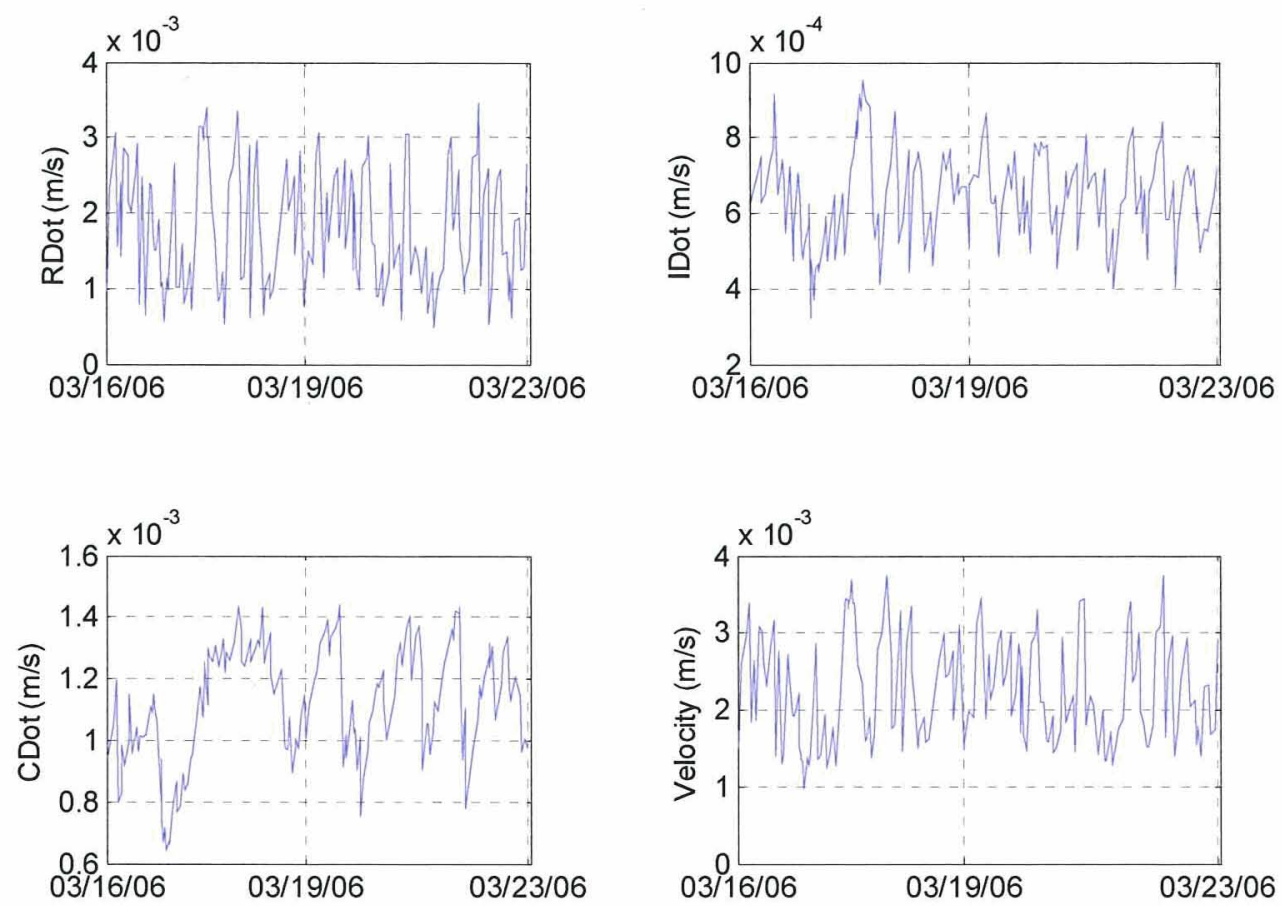

Figure 2: Definitive Velocity Root-Variance

After the 'averaged' $6 \times 6$ position and velocity covariance is constructed, an initial $C_{d}$ variance of 0.01 is assumed. Then the final form of $P\left(t_{0}\right)$ is given as follows, where units of the $6 \times 6$ position/velocity covariance are $\mathrm{m}^{2}$ and $(\mathrm{m} / \mathrm{s})^{2}$ and the $C_{d}$ sigma is unitless:

$$
P\left(t_{0}\right)=\left(\begin{array}{ccccccc}
0.4968374877 & 0.0675614206 & -0.1441904874 & -0.0000349037 & -0.0005172160 & 0.0000743303 & 0 \\
0.0675614206 & 6.3756755001 & -0.0011264630 & -0.0062663352 & -0.0002362080 & 0.0000543959 & 0 \\
-0.1441904874 & -0.0011264630 & 0.8830755578 & -0.0001093147 & 0.0001535472 & 0.0001868132 & 0 \\
-0.0000349037 & -0.0062663352 & -0.0001093147 & 0.0000065510 & 0.0000002171 & -0.0000003099 & 0 \\
-0.0005172160 & -0.0002362080 & 0.0001535472 & 0.0000002171 & 0.0000005480 & -0.0000000837 & 0 \\
0.0000743303 & 0.0000543959 & 0.0001868132 & -0.0000003099 & -0.0000000837 & 0.0000010855 & 0 \\
0 & 0 & 0 & 0 & 0 & 0 & 0.01
\end{array}\right)
$$

\section{F. C. Prediction Error Characterization}

Next the measured position prediction errors are characterized by applying the method described in Section II-B. First, for each RIC component, the RMS statistic is calculated for prediction times up to 7 days. A least-squares polynomial fit to the RMS statistics is performed. Figure 3 shows the prediction error information for Aura. The blue squares on the left hand side are the measured RMS error statistics collected at 6 -hour increments. The red line is the prediction error 'best-fit' polynomial $\sigma_{i}^{M}$. On the right hand side are the fit residuals, which are denoted by 'plus' signs. The residuals are the differences between the measured RMS prediction error and the polynomial fit. 

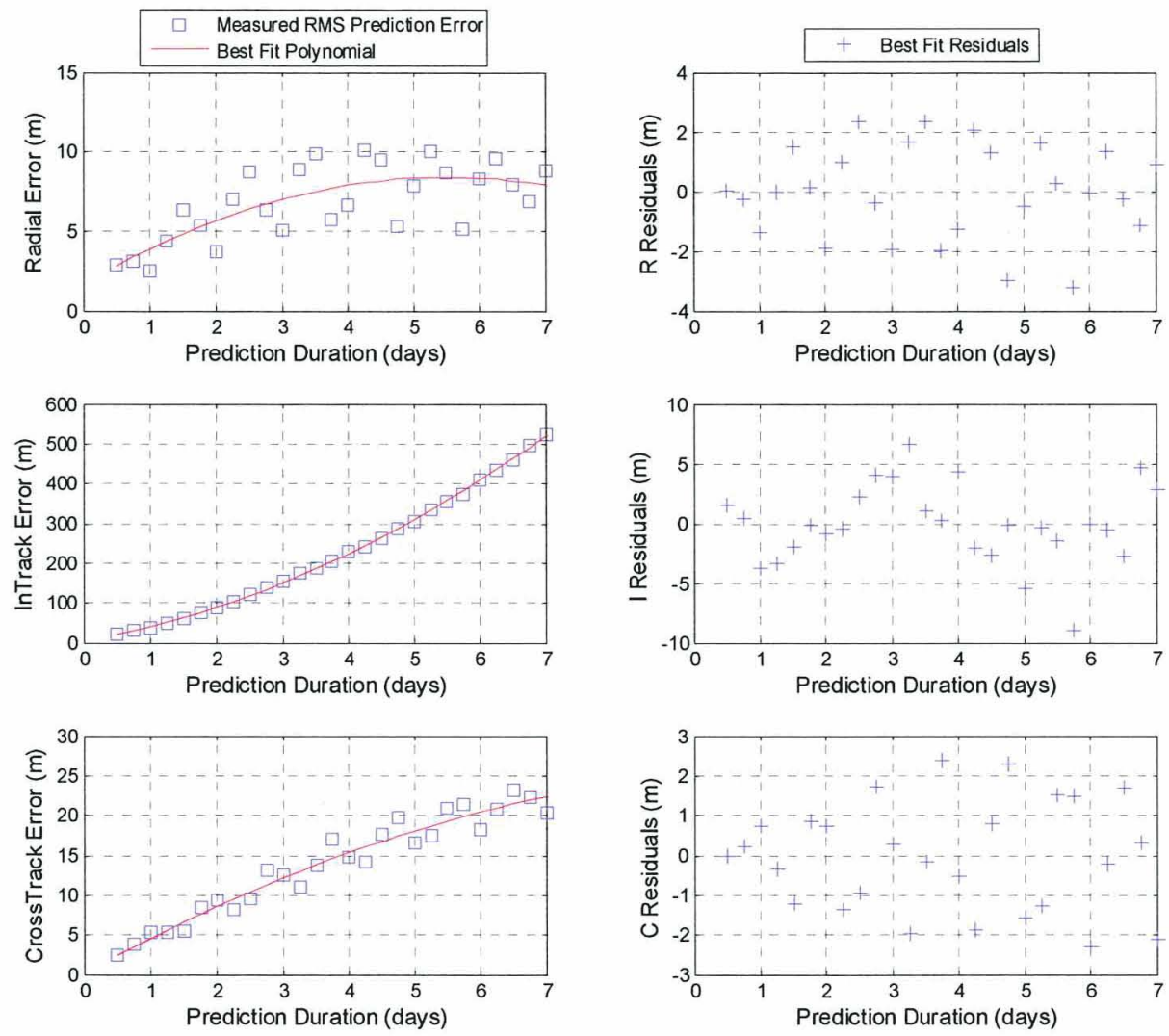

Figure 3: Measured Position Prediction Errors

The prediction error statistics were generated using data spanning January 01, 2006 - March 10, 2006. Figure 3 shows that the dominant error is in the in-track direction, where the measured error grows to approximately $150 \mathrm{~m}$ after 3 days and approaches $600 \mathrm{~m}$ after 7 days. The measured cross-track error grows from 5 to 25 meters over the prediction span; the radial error is equal to about one half of the crosstrack error. Table 4 below lists the prediction error polynomials that are plotted in Figure 3.

Table 4: Prediction Error Polynomials

\begin{tabular}{|l|c|}
\hline Position Component & Measured Prediction Error \\
\hline Radial (meters) & $\sigma_{R}^{M}=-0.222 t^{2}+2.444 t+1.668$ \\
\hline In-Track (meters) & $\sigma_{I}^{M}=6.217 t^{2}+29.851 t+5.747$ \\
\hline Cross-Track (meters) & $\sigma_{C}^{M}=-0.203 t^{2}+4.589 t+0.246$ \\
\hline
\end{tabular}

\section{Predictive Covariance Calibration - Aura}

After the prediction errors are characterized, comparisons are made between the measured error $\left\{\sigma_{R}^{M}, \sigma_{I}^{M}, \sigma_{C}^{M}\right\}$ and the root-variance of the covariance prediction $\left\{\sigma_{R}^{P}, \sigma_{I}^{P}, \sigma_{C}^{P}\right\}$. The predictive covariance is 'calibrated' so that the differences between $\sigma_{i}^{M}$ and $\sigma_{i}^{P}$ are sufficiently small for each position component.

To illustrate the need for the addition of the process noise matrix $Q$, Figure 4 shows the results of a comparison between the measured position error and the prediction root-variance without the addition of the process noise matrix. That is, $P\left(t_{0}\right)$ is propagated forward using $P(t)=\Phi\left(t, t_{0}\right) P\left(t_{0}\right) \Phi\left(t, t_{0}\right)^{T}$.

American Institute of Aeronautics and Astronautics 

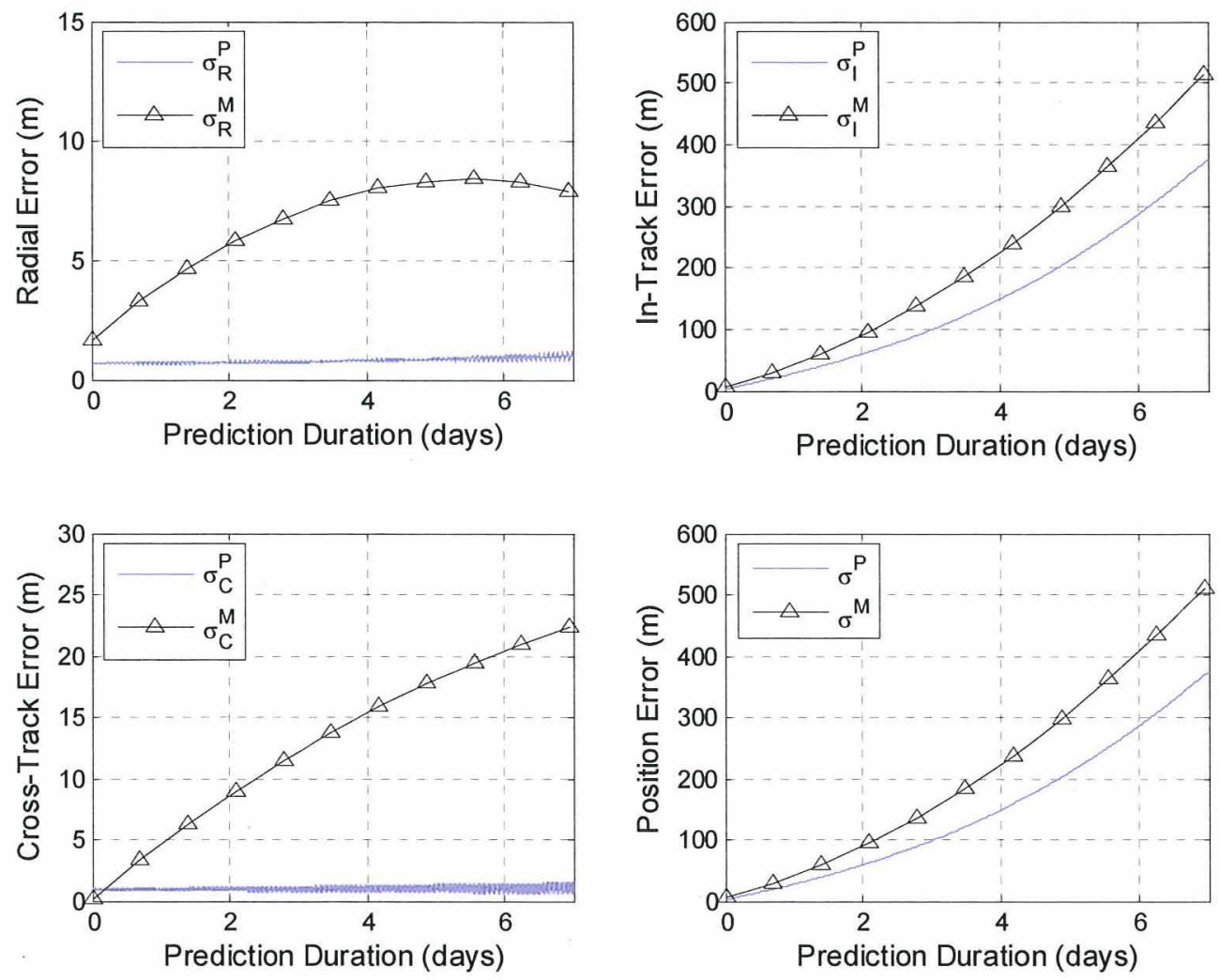

Figure 4: RIC Position Error Predictions without Process Noise

In each of the four graphs, the lower of the two error curves is the prediction root-variance that was obtained by propagating $P\left(t_{0}\right)$ forward in time without process noise. Examination of Figure 4 clearly shows that each root-variance $\left\{\sigma_{R}^{P}, \sigma_{I}^{P}, \sigma_{C}^{P}\right\}$ of $P(t)$ underestimates the measured error $\left\{\sigma_{R}^{M}, \sigma_{I}^{M}, \sigma_{C}^{M}\right\}$ as $P$ is propagated forward. There is virtually no error growth in either the radial or the cross-track components. Additionally, the difference between the two in-track error predictions grows to approximately $50 \mathrm{~m}$ after 3 days and nearly $100 \mathrm{~m}$ after 5 days. Therefore, in the absence of process noise, the predicted covariance matrix provides a conservative or 'optimistic' representation of the position uncertainty.

As described in Section II-C, the process noise calibration methodology consists of: examining the rootvariance of the predicted covariance; comparing the predicted covariance to the measured prediction error; and making adjustments to $Q$ as necessary so that the two predictions are in good agreement. Since values of $\sigma_{\ddot{I}}$ and $Q_{C_{d}}$ both affect the in-track prediction error, it is sufficient to choose to vary only one when adjusting the in-track error growth. So $Q_{C_{d}}$ is set to zero and the prediction convergence is chosen such that, over the entire prediction span, the radial and cross-track differences are within $5 \mathrm{~m}$ and the in-track differences are within $10 \mathrm{~m}$. That is, for all prediction times $t$,

$$
\left|\sigma_{R}^{M}-\sigma_{R}^{P}\right|<5 \quad\left|\sigma_{I}^{M}-\sigma_{I}^{P}\right|<10 \quad\left|\sigma_{C}^{M}-\sigma_{C}^{P}\right|<5
$$

Equation 12 is satisfied with the following RIC acceleration variances: 


$$
\bar{q}_{a c c}=\left(\begin{array}{c}
1.4 e-9 \\
2.4 e-11 \\
5 e-9
\end{array}\right)^{2}
$$

Figure 5 and Figure 6 show the results of adding the process noise $Q$-matrix to the predictive covariance generation. Each plot in Figure 5 contains two curves; namely $\sigma_{i}^{M}$ and $\sigma_{i}^{P}$ for each position component and the root-sum-square of the position components $\sigma^{M}$ and $\sigma^{P}$.
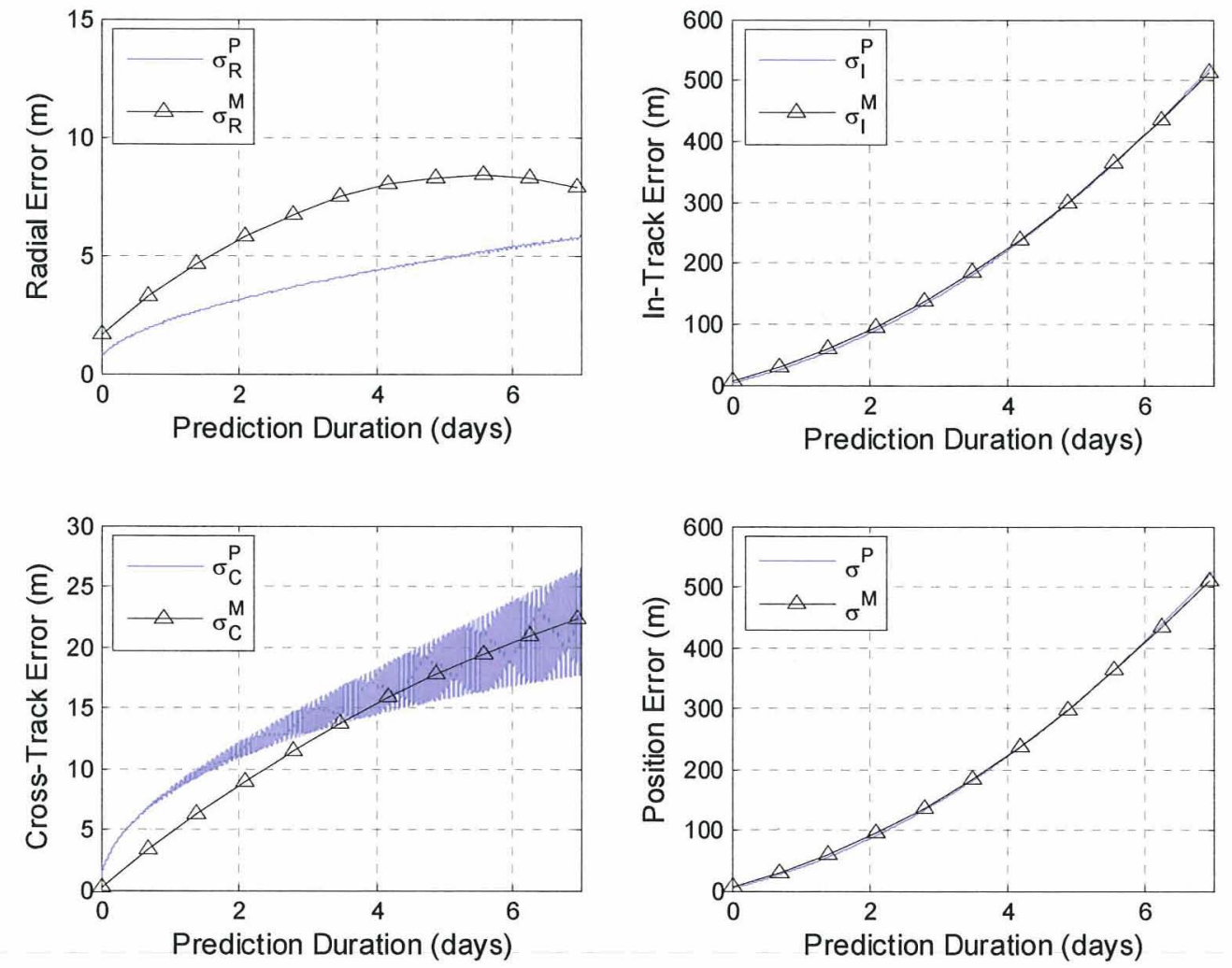

Figure 5: Position Error Predictions with Process Noise

The differences between the two error predictions are shown in Figure 6. The radial and cross-track differences remain within the specified $5 \mathrm{~m}$ convergence threshold; the in-track difference stays within 10 $\mathrm{m}$. This gives a total position error difference of $10 \mathrm{~m}$ for the entire prediction time $t$. 

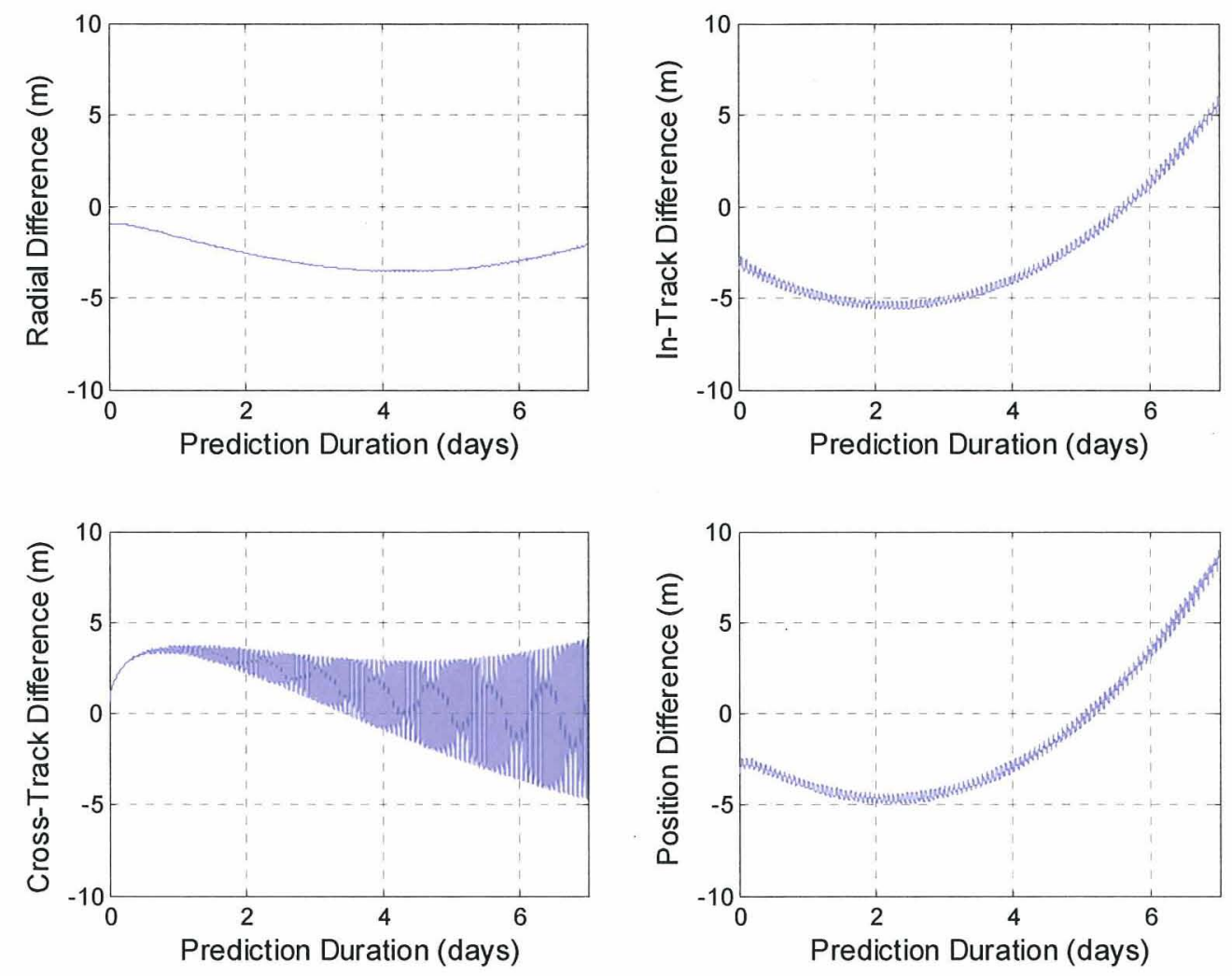

Figure 6: Position Prediction Error Differences

\section{Conclusions and Future Work}

The collision risk assessment process requires realistic state uncertainty information to make accurate collision probability calculations. A three-step methodology describing realistic covariance predictions has been introduced. This method is straightforward and has been used to construct covariance prediction models for the Aqua, Aura, and Terra missions. In the future, this method will be enhanced to include the generation of realistic covariance predictions through periods containing spacecraft maneuvers.

\section{References}

1. Newman, L.K, and Duncan, M., "Establishment and Implementation of a Close Approach Evaluation and Avoidance Process for the Earth Observing System Missions" AIAA Paper 06-xxx.

2. Alfriend, K.T., and Wilkins, M.P., "Covariance as an Estimator of Orbit Prediction Error Growth in the Presence of Unknown Sensor Biases," AAS paper 99-422.

3. Alfriend, K.T., France, R., Glover, R., and Hoots, F., "Single Track Covariance for Space Surveillance Network Sensors," AAS paper 98-115.

4. Montenbruck, O., and Gill, E., Satellite Orbits, Springer-Verlag Berlin Heidelberg New York, 2001, pg. 263-266.

5. Barker, W., Casali, S., and Walker, C., "Improved Space Surveillance Network Observation Error Modeling and Techniques for Force Model Error Mitigation," AAS 99-420. 